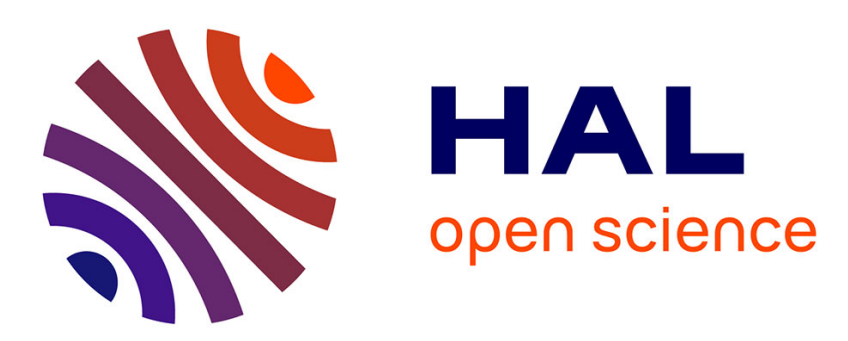

\title{
Une meilleure gestion patrimoniale des réseaux d'eau potable: le modèle de prévision du renouvellement à long terme OPTIMEAU
}

Aurore Large

\section{> To cite this version:}

Aurore Large. Une meilleure gestion patrimoniale des réseaux d'eau potable : le modèle de prévision du renouvellement à long terme OPTIMEAU. Rencontres Universitaires de Génie Civil, May 2015, Bayonne, France. hal-01167672

\section{HAL Id: hal-01167672 \\ https://hal.science/hal-01167672}

Submitted on 24 Jun 2015

HAL is a multi-disciplinary open access archive for the deposit and dissemination of scientific research documents, whether they are published or not. The documents may come from teaching and research institutions in France or abroad, or from public or private research centers.
L'archive ouverte pluridisciplinaire HAL, est destinée au dépôt et à la diffusion de documents scientifiques de niveau recherche, publiés ou non, émanant des établissements d'enseignement et de recherche français ou étrangers, des laboratoires publics ou privés. 


\title{
Une meilleure gestion patrimoniale des réseaux d'eau potable : le modèle de prévision du renouvellement à long terme OPTIMEAU
}

\author{
LARGE Aurore ${ }^{1,2}$ \\ ${ }^{1}$ Université de Bordeaux, I2M - Institut de Mécanique et d'Ingénierie, UMR CNRS 5295, 351 cours de la Libéra- \\ tion, 33405 Talence Cedex, aurore.large@u-bordeaux.fr \\ ${ }^{2}$ IRSTEA - Institut national de Recherche en Sciences et Technologies pour l'Environnement et l'Agriculture, \\ UR ETBX - Environnement Territoires et Infrastructures Bordeaux, 50 avenue de Verdun, 33612 Cestas, au- \\ rore.large@irstea.fr
}

Prix Jeunes Chercheurs «René Houpert »

RÉSUMÉ. Dans les pays développés, l'eau potable est distribuée au domicile des usagers. Ce confort requiert un long linéaire de réseau de forte valeur patrimoniale. Pour optimiser ressources et performances, il est important d'essayer de renouveler les tronçons au meilleur moment possible. Cet article présente les modèles court (1 à 3 ans), moyen et long terme ( > 30 ans) actuellement employés. Les processus court terme semblent assez efficaces, mais les méthodes moyen et long terme restent frustes. Le modèle OPTIMEAU propose une approche long terme pour obtenir une vision globale du patrimoine de canalisations et rationaliser sa gestion, tout en restant en phase avec les pratiques opérationnelles de programmation à court terme. Cette démarche, fondée sur des modèles statistiques de survie, est testée à partir des données réelles de 3 services d'eau Européens : le SEDIF (Syndicat des Eaux d'Ile de France), le Grand Lyon et eauservice Lausanne en Suisse. L'originalité de ce modèle réside dans l'estimation de la distribution des âges à la mise hors service passés, clef de voûte dans la construction de onze indicateurs liés aux finances, aux réalisations et à la performance future des services.

ABSTRACT. In developed countries, drinking water is distributed to households. This comfort requires a long network with a high value. To optimize resources and performance, it is important to renew pipe sections at the best possible time. This article presents the short (1-3 years), medium and long term (> 30 years) models commonly employed. The short-term processes seem quite effective, but long term methods remain rough. The OPTIMEAU model proposes a long-term approach to get a prospective overview of pipes and improve its management, while remaining connected to the technical practices at short-term level. This approach, based on survival statistical models, is tested using actual data of three European water services : SEDIF (Syndicat des Eaux d'Ile de France), Grand Lyon and eauservice Lausanne in Switzerland. The originality of this model lies in the estimation of the past decommissioning age distribution, keystone in the construction of eleven indicators related to finance, achievements and future performance of water utilities.

MOTS-CLÉS : : remplacement, optimisation, coûts-performance, risque, survie, canalisation d'eau potable

KEYWORDS: : renewal, optimization, cost-effective, risk, survival, drinking water pipe 


\section{Introduction}

Dans les pays développés, l'eau potable est distribuée au domicile des usagers. Ce confort implique un long linéaire de canalisations avec une valeur élevée. En France actuellement ce réseau mesure plus de $1050000 \mathrm{~km}$ [DEQ 14] avec une valeur d'environ 152 milliards d'euros [OIE 12]. Le renouvellement annuel d'une fraction du linéaire des canalisations est nécessaire du fait de l'usure normale de ces objets techniques, qui entraîne leur mauvais état et la diminution progressive de leur niveau de performance. En parallèle de l'usure, des opportunités ou des contraintes (coordination avec d'autres domaines de l'ingénierie urbaine, aménagement du territoire, législation plus exigeante etc.) peuvent conduire au remplacement de certaines canalisations bien que leur état soit satisfaisant. Dans une optique d'optimisation des ressources et des performances, il est essentiel de renouveler les tronçons de canalisation au meilleur moment possible, évitant ainsi des travaux trop précoces ou curatifs (après de trop nombreuses défaillances et de sévères dégâts). Une bonne gestion patrimoniale du réseau d'eau doit donc prendre en compte cet enjeu.

Une fois que le service d'eau a fixé ses propres objectifs, une gestion patrimoniale de bonne qualité doit enchaîner cycliquement les quatre grandes phases de la roue de Deming : 1) la planification des actions 2) leur réalisation 3) leur évaluation puis 4) leur réajustement si elles n'ont pas permis d'atteindre les objectifs initiaux. Cette démarche doit aboutir à des améliorations en continue.

Actuellement les services d'eau utilisent 3 échelles de temps pour la planification de leurs actions : le court terme ( 1 à 3 ans à venir), le moyen terme (5 à 20 ans) et le long terme (30 à 170 ans à venir). Dans les prévisions à court terme, le budget est fixe et les modèles permettent d'obtenir une liste de tronçons hiérarchisés. Le moyen terme est l'échelle de construction des plans pluriannuels d'investissement (PPI) des services d'eau. Par exemple à Lausanne le PPI est de 10 ans, au Grand Lyon le PPI est de 6 ans et au SEDIF le PPI dure 5 ans. Enfin le long terme est l'échelle des stratégies patrimoniales. A cette échelle plusieurs stratégies peuvent être définies et testées, puis, en comparant, les réalisations et les résultats prévus pour chaque stratégie aux objectifs du service, une stratégie peut être choisie.

Pour optimiser ressources et performance il semble logique de partir d'une vision long terme permettant de choisir une stratégie pour respecter les objectifs en termes de performances du service d'eau, puis d'en déduire le besoin en investissement à moyen terme. Il faut ensuite appliquer les méthodes de court terme permettant d'obtenir notamment pour l'année à venir une liste de tronçons hiérarchisés par niveau de besoin et opportunités en renouvellement, en s'assurant que ces opérations permettent effectivement d'atteindre les performances visées.

L'analyse approfondie des modèles décisionnels existants à ces 3 échelles de temps [LAR 13] a montré que les méthodes décisionnelles long terme sont souvent inexistantes ou déconnectées des méthodes décisionnelles de moyen terme et de court terme.

La revue bibliographique et les enquêtes de terrain ont mis en évidence 3 familles de modèles prévisionnel à long terme.

La première famille de modèles (cf. [JAR 01], [SAE 03], [ENG 05], [MAR 09]) est basée sur un seuil de défaillances que les gestionnaires ne souhaitent pas dépasser. Cependant, lorsqu'on a demandé aux services d'eau quels étaient leurs propres seuils, ils n'ont pas su réponde. En effet, en général le nombre de défaillances n'est pas l'indicateur qu'ils utilisent pour se fixer des objectifs mais ils utilisent plutôt le niveau de dommages que peuvent induire certaines défaillances. Par conséquent, il a été décidé d'écarter cette famille de modèles.

La seconde famille de modèles (cf. Cador [CAD 02], Patrimoine Expert [NAL 13]) se résument simplement à additionner une durée de maintien en service hypothétique à la date de pose du tronçon pour obtenir la date de mise hors service présumée. Cette démarche ne convainc pas les praticiens. En effet elle fait l'hypothèse que les canalisations doivent être remplacées à âge fixe, ce qui est non pertinent : en fonction des contraintes que subit une canalisation, certaines très âgées peuvent être en bon état et d'autres très jeunes en mauvais état. De plus aucun lien n'est fait entre cette durée de maintien en service hypothétique et le processus décisionnel opérationnel de moyen et court terme (coordination, risques, etc.), dont résulte effectivement l'âge à la mise hors service.

La troisième famille de modèles parait la plus prometteuse (cf. Kanew [HER 02], [KRO 13], Nessie [AWW 10], PiREM [FUC 08]). Elle suppose que les canalisations ne sont pas renouvelées à âge fixe mais selon une distribution statistique (courbes en cloche) de l'âge à la mise hors service. Néanmoins ces modèles allemand, australien et autrichien ne sont utilisés par aucun service d'eau en France. La faiblesse majeure de ces modèles est que la distribution des âges à la mise hors service n'est pas issue des données des services d'eau, mais, elle aussi établie a priori à partir d'hypothèses souvent très grossières. La question de la représentativité et de la pertinence de ces courbes de survie par rapport aux données du service, à son processus de décision moyen et court terme passé, et à ses objectifs futurs demeure entière. 
Cet article présente le résultat d'une réflexion menée sur ce problème. Cette réflexion a débouché sur la proposition d'une nouvelle méthode long terme fiable permettant d'aider les gestionnaires dans le choix de leurs réalisations (linéaire, matériaux, etc.), en lien avec leur propre processus décisionnel de moyen et court terme et de leurs objectifs de performance. Cette méthode s'inspire de la troisième famille de modèle présentée précédemment. Les deux principales innovations de la méthode proposée OPTIMEAU résident dans le fait que 1) la distribution des âges à la mise hors service passé n'est pas hypothétique mais dépend des pratiques passées du service d'eau 2) la prévision du nombre de défaillances futures (et des risques) est réalisée à l'échelle du tronçon à l'aide du modèle statistico-probabiliste de LEYP (Linear Extented Yule Process) [LEG 13] et non selon une équation linéaire arbitraire.

\section{Vue d'ensemble du modèle long terme OPTIMEAU}

Le travail a été mené en collaboration étroite avec trois grands services d'eau européens : le Syndicat des Eaux d'Ile de France (SEDIF), la communauté urbaine de Lyon (Grand Lyon) et le Service des Eaux de Lausanne (eauservice) dont les linéaires respectifs sont de $8300 \mathrm{~km}, 3900 \mathrm{~km}$ et $900 \mathrm{~km}$. La démarche globale du modèle long terme OPTIMEAU peut se décomposer en 4 grandes étapes.

La première étape comprend l'analyse de la politique de gestion patrimoniale que le service d'eau a mené par le passé, afin de répondre notamment à la question suivante : quelles ont été les causes de mise hors service des tronçons? Au cours de cette étape est calculée la distribution des âges à la mise hors service sur une fenêtre d'observation passée. Ce calcul peut être effectué sur tout le réseau ou par groupe de tronçons (strate) en fonction de leur matériau et/ou leur diamètre etc. Il est à noter que tous les calculs dans ce modèle sont effectués à partir de la fonction de survie des âges à la mise hors service. Cette fonction est tout simplement le complémentaire à l'unité de l'intégrale de la distribution des âges à la mise hors service.

La seconde étape consiste à élaborer différents scénarios prospectifs. Associés à ces scénarios le gestionnaire doit identifier ses objectifs, des indicateurs et des seuils (planchers ou plafonds). Par exemple le scénario prospectif «statu quo » peut être : «A l'avenir la distribution des âges à la mise hors service des canalisations sera strictement identique à celle par le passé entre [2002, 2012] ». Néanmoins les scénarios prospectifs peuvent être plus complexes. Un exemple de scénario possible est : «Les canalisations en PVC (Polychlorure de Vinyle) posées avant 1980 et celles en amiante ciment doivent être éliminées avant 2020. Les canalisations ayant une forte probabilité d'induire des dommages considérables suite à leurs défaillances seront renouvelées en priorité. La part des canalisations renouvelées sous la contrainte (par exemple, coordination avec les chantiers du tramway) sera plus faible que par le passé. A l'avenir toutes les canalisations seront en fonte ductile. » Ensuite, chaque scénario prospectif est traduit par une fonction de survie des âges à la mise hors service prospective pour chaque strate. Cela consiste en la transformation des fonctions de survie obtenues à l'étape 1 .

Lors de l'étape trois, pour chaque scénario, onze indicateurs prévisionnels annuels sont déduits à partir des courbes de survie obtenues à l'étape précédente. Il s'agit de 2 indicateurs de moyens financiers, 3 indicateurs des réalisations et 6 indicateurs de résultats (ou de performance) (cf. la liste complète portée à la figure 1).

Enfin au cours de l'étape quatre on compare les différents scénarios par le biais des valeurs des onze indicateurs calculés à l'étape 4. En fonction de ses objectifs et contraintes (budgétaires ou autres), le gestionnaire du service d'eau peut alors choisir le scénario qu'il souhaite appliquer, étant averti de ses conséquences (mises en évidence par les 11 indicateurs). Le modèle OPTIMEAU fournit un tableau de bord avec ces indicateurs et la collectivité en dispose comme elle le souhaite.

\section{Détail de la levée de quatre verrous majeurs du modèle OPTIMEAU}

Afin de mener de bout en bout la méthode, le calcul des différents indicateurs énoncés à la figure 1 nécessite de lever quatre verrous opérationnels : (1) l'estimation des courbes de survie passées sur une fenêtre d'observation, (2) la prévision du linéaire à renouveler chaque année I1R, (3) la prédiction du nombre de défaillances futures sur le long terme I1P, et enfin (4) le calcul des indicateurs de risque liés aux défaillances.

\subsection{Estimation des fonctions de survie rétrospectives}

L'étape 1 requiert l'estimation des courbes de survie passées sur une fenêtre d'observation. La fonction de survie notée $S$ est la probabilité $\mathrm{P}$ que l'âge à la mise hors service $T$ d'un groupe de tronçons (ou strate) soit supérieur à un certain âge $t: S(t)=\mathrm{P}(T>t)$. 


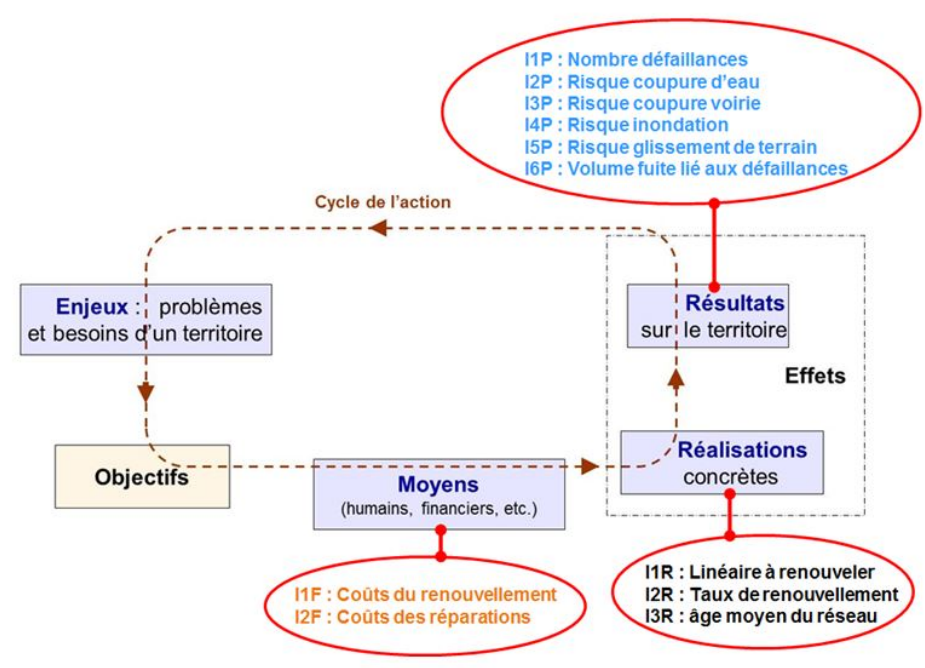

Figure 1. Les onze indicateurs du modèle OPTIMEAU et leurs localisations dans le cycle de l'action (F pour financier, $R$ pour réalisation et $P$ pour performance).

Une fenêtre d'observation, notée $\left[y_{1}, y_{2}\right]$ est une période pendant laquelle les dates de mise hors service et les dates de défaillances des tronçons ont été enregistrées au sein du service d'eau de manière exhaustive. $y_{1}$ est l'année de début de la fenêtre d'observation (ex : 1995 au SEDIF) et $y_{2}$ est l'année de fin de la fenêtre d'observation (ex : 2012 au SEDIF). Sur cette fenêtre d'observation, chaque tronçon $i$ est caractérisé par : sa longueur $L_{i}$, son âge au début de son observation dans la fenêtre $t_{1 i}$, son âge à la fin de son observation dans la fenêtre $t_{2 i}$ et sa censure $c_{i}$. La censure est une variable indicatrice de valeur 1 si le tronçon est encore en service à la fin de la fenêtre d'observation et de valeur 0 si le tronçon a été mis hors service dans la fenêtre d'observation. Pour estimer correctement la survie passée noté $\widehat{S}_{\mathrm{K}}(t)$ on s'est inspiré des méthodes employées par les épidémiologistes (voir Kaplan et Meier [KAP 58]) (cf. équation 1).

$$
\widehat{S}_{\mathrm{K}}(t)=\prod_{a \leq t}\left[1-\left(\sum_{i: t_{2 i}=a, c_{i}=0} L_{i}\right) /\left(\sum_{i: t_{1 i} \leq a, t_{2 i} \geq a} L_{i}\right)\right]
$$

Une fois les scénarios prospectifs élaborés, au cours de l'étape 2, il est nécessaire de leur associer une fonction de survie prospective continue. Plusieurs méthodes ont été testées. Par exemple pour le scénario statu quo, la fonction de survie de Weibull [WEI 51] noté $\widehat{S}_{\mathrm{W}}(t)$ de paramètres $\eta$ et $\gamma\left(\widehat{S}_{\mathrm{W}}(t)=\mathrm{e}^{-(t / \eta)^{\gamma}}\right)$ a été ajustée par la méthode des moindres carrés à $\widehat{S}_{\mathrm{K}}(t) . \widehat{S}_{\mathrm{W}}(t)$ est alors dans ce cas la survie prospective.

\subsection{Prévision du linéaire à renouveler}

A l'étape 3 les indicateurs I1P et I1R sont les clés de voûte pour le calcul des autres indicateurs. Le calcul du linéaire à renouveler année par année I1R est effectué à l'échelle de la cohorte. Une cohorte est composée de tous les tronçons posés l'année $y_{0}$. Chaque cohorte est caractérisée par sa longueur encore en service l'année $y$, notée $L E_{y_{0}}(y)$, sa longueur ayant été mis hors service entre l'année $y_{0}$ et l'année $y$, notée $L H_{y_{0}}(y)$ et sa survie à l'âge $t=y-y_{0}$, notée $S\left(y-y_{0}\right)$. La première année de simulation prospective est notée $y_{2}+1$ (ex : 2013 au SEDIF). Notons que $\operatorname{I1R}(y)=L E_{y_{0}}(y)+L H_{y_{0}}(y)$

$$
\begin{gathered}
L E_{y_{0}}\left(y_{2}+1\right)=L E_{y_{0}}\left(y_{2}\right) \times \frac{S\left(y_{2}+1-y_{0}\right)}{S\left(y_{2}-y_{0}\right)} \\
L E_{y_{2}+1}\left(y_{2}+1\right)=\sum_{y_{0}=\min \left(y_{0}\right)}^{y_{2}}\left[L E_{y_{0}}\left(y_{2}\right)-L E_{y_{0}}\left(y_{2}+1\right)\right]
\end{gathered}
$$




$$
L H_{y_{0}}(y)=L E_{y_{0}}\left(y_{0}\right)-L E_{y_{0}}(y)
$$

Le calcul d'I1P nécessite d'enchaîner les équations 2,3 et 4, puis d'itérer année par année jusqu'à obtenir l'année prospective désirée (par exemple 2050 au SEDIF).

\subsection{Prédiction du nombre de défaillances}

La première phase nécessaire au calcul du nombre de défaillances prévisionnel (I1P) consiste en la transformation de la fonction de survie prospective en matrice de transition de Markov [MAR 10] notée $M$ (cf. équation 5). Elle contient dans chaque cellule la probabilité de passer d'un âge $t$ (ligne) entre $\left[1 ; t_{\max }\right]$ à un autre âge (colonne) entre $\left[1 ; t_{\max }\right]$, avec $t_{\max }$ l'âge maximal que peut avoir un tronçon. Par exemple dans les équations 5,6 et 8 , $t_{\max }=150$ ans comme à Lausanne. La matrice $M$ est originale car elle concerne l'âge des conduites et non pas l'état physique, comme on le voit plus traditionnellement.

$$
\boldsymbol{M}=\left[\begin{array}{cccccccc}
1-\frac{S(1)}{S(0)} & \frac{S(1)}{S(0)} & 0 & 0 & 0 & 0 & 0 & 0 \\
1-\frac{S(2)}{S(1)} & 0 & \frac{S(2)}{S(1)} & 0 & 0 & 0 & 0 & 0 \\
\cdots & 0 & 0 & \cdots & 0 & 0 & 0 & 0 \\
1-\frac{S(t)}{S(t-1)} & 0 & 0 & 0 & \frac{S(t)}{S(t-1)} & 0 & 0 & 0 \\
\cdots & 0 & 0 & 0 & 0 & \cdots & 0 & 0 \\
1-\frac{S(148)}{S(147)} & 0 & 0 & 0 & 0 & 0 & \frac{S(148)}{S(147)} & 0 \\
1-\frac{S(149)}{S(148)} & 0 & 0 & 0 & 0 & 0 & 0 & \frac{S(149)}{S(148)} \\
1 & 0 & 0 & 0 & 0 & 0 & 0 & 0
\end{array}\right]
$$

La seconde phase consiste à obtenir pour chaque tronçon lieu $i$ un vecteur contenant les probabilités d'avoir un âge (entre 1 et $t_{\max }$ ) l'année $y$ noté $\boldsymbol{A}_{i}^{\prime}(y)$. Un tronçon lieu est un tronçon qui garde les mêmes coordonnées $(x, y, z)$ que le tronçon physique de l'année $y_{2}$ néanmoins son âge, son matériau et son diamètre peuvent changer au cours du temps lors des différents renouvellements des tronçons physiques. Le tronçon lieu conserve donc sur du long terme sa longueur et ses autres variables (distance au réservoir, corrosivité du sol, etc.). $y$ varie de $y_{2}$ jusqu'à l'année de fin de la simulation prospective notée $y_{2}+k(k \in \mathbb{N})$. $\boldsymbol{A}_{i}^{\prime}\left(y_{2}\right)$ est définie selon l'équation 6 , puis en utilisant la théorie de la chaîne de Markov [MAR 10] $\boldsymbol{A}_{i}^{\prime}\left(y_{2}+k\right)$ est obtenue grâce à l'équation 7 .

$$
\begin{aligned}
\boldsymbol{A}_{i}^{\prime}\left(y_{2}\right)= & \left.\begin{array}{ccccccc}
1 & 2 & \ldots & y_{2}-y_{0} & \ldots & 149 & 150 \\
0 & 0 & \ldots & 1 & \ldots & 0 & 0
\end{array}\right] \\
\boldsymbol{A}_{i}^{\prime}\left(y_{2}+k\right)= & \boldsymbol{A}_{i}^{\prime}\left(y_{2}\right) \times \boldsymbol{M}^{k}
\end{aligned}
$$

La troisième phase consiste à déterminer une équation de prédiction de la détérioration des tronçons physiques au cours du temps. Lors de cette phase il a été décidé d'utiliser le modèle statistico-probabiliste de LEYP [LEG 13] qui semble être le plus fiable de sa génération. Le modèle de LEYP est un modèle de régression stastistique qui permet d'estimer, de manière empirique, les défaillances futures en fonction de plusieurs variables du tronçon physique notamment : l'âge, le nombre de défaillances passées, le matériau, le diamètre, la pression de l'eau, le niveau de trafic routier, etc.

Issues du modèle de LEYP, les prévisions pour chaque tronçon physique $i$ de l'espérance du nombre de défaillances annuel à l'âge $t$ notée $\frac{\mathbb{E}_{\theta}\left[\mathrm{d} N_{i}(t)\right]}{\mathrm{d} t}$ sont stockées dans le vecteur $\boldsymbol{D}_{i}$ (cf. équation 8).

$$
\boldsymbol{D}_{i}=\left[\mathbb{E}_{\boldsymbol{\theta}}\left[\mathrm{d} N_{i}(t)\right] / \mathrm{d} t\right]_{1 \leq t \leq 150}
$$

La quatrième phase consiste à croiser les âges probables des tronçons avec les résultats de la prévision du processus de la détérioration afin d'obtenir l'espérance du nombre de défaillances pour chaque tronçon lieu $i$ l'année $y$ noté $\Psi_{i}(y)$ (cf. équation 9). Enfin pour obtenir le nombre de défaillance sur le réseau notée $\Psi(y)$ il suffit de sommer pour chaque tronçon les $\Psi_{i}(y)$ (cf. équation 9). Notons qu' $\operatorname{I1P}(y)=\Psi(y)$.

$$
\Psi(2012+k)=\sum_{i} \Psi_{i}(2012+k)=\sum_{i}\left[\boldsymbol{A}_{i}^{\prime}(2012+k) \times \boldsymbol{D}_{i}\right]
$$




\subsection{Détermination des indicateurs de risques de dommages liés aux défaillances}

Un dommage est toujours issu de la rencontre d'un danger, ici une défaillance, avec des éléments vulnérables, par exemple les usagers sensibles aux coupures d'eau [CHE 07]. Les indicateurs de risque dans le modèle long terme OPTIMEAU sont toujours construits de la même manière. Le niveau de gravité du risque de dommage est égal au produit de la probabilité de la défaillance et de son intensité par le nombre d'éléments vulnérables, leur vulnérabilité et leur valeur. Par exemple le niveau de risque de dommage lié à une coupure d'eau induit par une défaillance sur le tronçon $i$ noté $R C O N_{i}(y)$ est égal au produit de la probabilité de défaillances du tronçon $\Psi_{i}(y)$ et du temps nécessaire pour effectuer la réparation de cette dernière noté $E D I_{i}$, par le nombre de personnes raccordées à ce tronçon noté $N P S_{i}$ et leur vulnérabilité $S C_{i}$. Pour obtenir ce risque à l'échelle du réseau $R C O N(y)$, il suffit de sommer les risques concernant chaque tronçon lieu $i$ (cf. équation 10). Notons qu'I2P $(y)=R C O N(y)$.

$$
R C O N(y)=\sum_{i} R C O N_{i}(y)=\sum_{i}\left[\Psi_{i}(y) \times E D I_{i} \times N P S_{i} \times S C_{i}\right]
$$

\section{Quelques sorties du modèle OPTIMEAU et discussions}

L'étape 1, concernant l'analyse des pratiques passées, s'effectue à l'échelle globale du réseau ou par groupe de tronçons (strates).

La figure 2 à gauche présente les résultats à l'échelle du réseau du SEDIF, du Grand Lyon et d'eauservice Lausanne. Les fenêtres d'observation de ces survies passées sont respectivement [1995, 2012], [2002, 2012] et [2001, 2012]. L'indice de fiabilité ${ }^{1}$ de ces courbes de survie, estimée à partir de la qualité des données d'entrée et aux erreurs du modèle, est respectivement égal à 99\%, 56\% et 96\%. Par conséquent les survies passées du SEDIF et d'eauservice Lausanne sont très fiables alors que celle du Grand Lyon l'est moyennement. On constate que la médiane de l'âge à la mise hors service des tronçons se situe à environ 56 ans pour eauservice Lausanne contre 102 ans pour le SEDIF. Ces valeurs sont tout à fait cohérentes avec les enquêtes de terrains sur les pratiques passées puisque eauservice, sur sa fenêtre d'observation, a un rythme de renouvellement autour de $1,4 \%$ par an contre $0,44 \%$ par an pour le SEDIF. Il est à noter que les indicateurs de performance de ces deux services sont tout à fait respectables, les pratiques sont juste différentes.

La figure 2 à droite présente les résultats pour le réseau de Lausanne stratifié par diamètre. L'indice de fiabilité de ces survies est de $100 \%$ pour les petits diamètres contre $95 \%$ pour les gros diamètres. On constate qu'eauservice Lausanne a eu tendance entre [2001, 2012] à plus renouveler les petits diamètres que les gros.

La figure 2 expose 5 exemples de survie passée. Notons qu'il est possible de stratifier sur beaucoup d'autres variable (le matériau, le type de joint, le niveau d'enfouissement, etc.) qui instruisent fortement sur les pratiques passés des services d'eau.
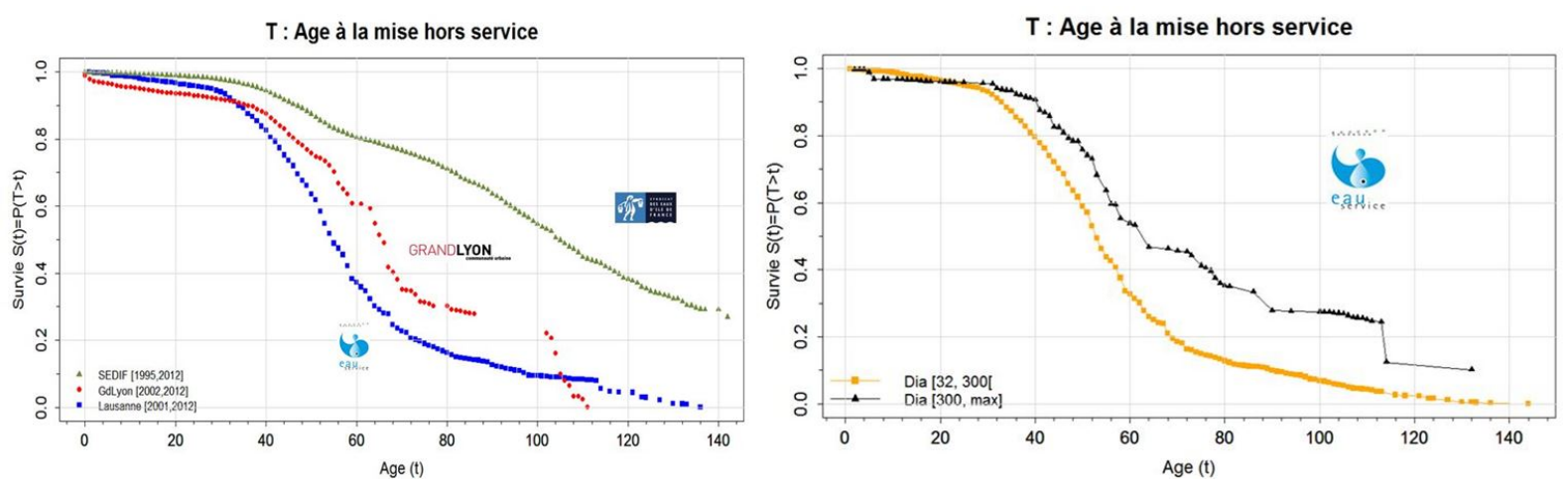

Figure 2. Courbes de survie passées du réseau du SEDIF, du Grand Lyon et d'eauservice Lausanne (à gauche) et du réseau d'eauservice Lausanne stratifié selon le diamètre (en $\mathrm{mm}$ ) (à droite).

A l'étape 2 c'est à dire lors de la construction de scénarios prospectifs et de survies prospectives une large palette de méthodes ont été testées. La plus simple consiste à appliquer le scénario statu quo. Ainsi pour le SEDIF, la survie de Weibull s'ajuste très bien (les p-values sont très inférieures à 5\%) : $\eta=130$ et $\gamma=2,3$.

1. Estimé par le croisement de l'intervalle de confiance de Rothman et des données manquantes en entrée du modèle 
Au cours de l'étape 3 onze indicateurs prévisionnels sont calculés. La figure 3 présente l'indicateur I1R sur le territoire du SEDIF avec le scénario statu quo pour une simulation démarrant en 2013 et terminant en 2050 en utilisant la fonction de survie de Weibull décrite précédemment. Cette fonction de survie induit des âges à la mise hors service tardifs donc les linéaires à renouveler chaque année ne sont pas très importants (autour de $0.6 \%$ par an).

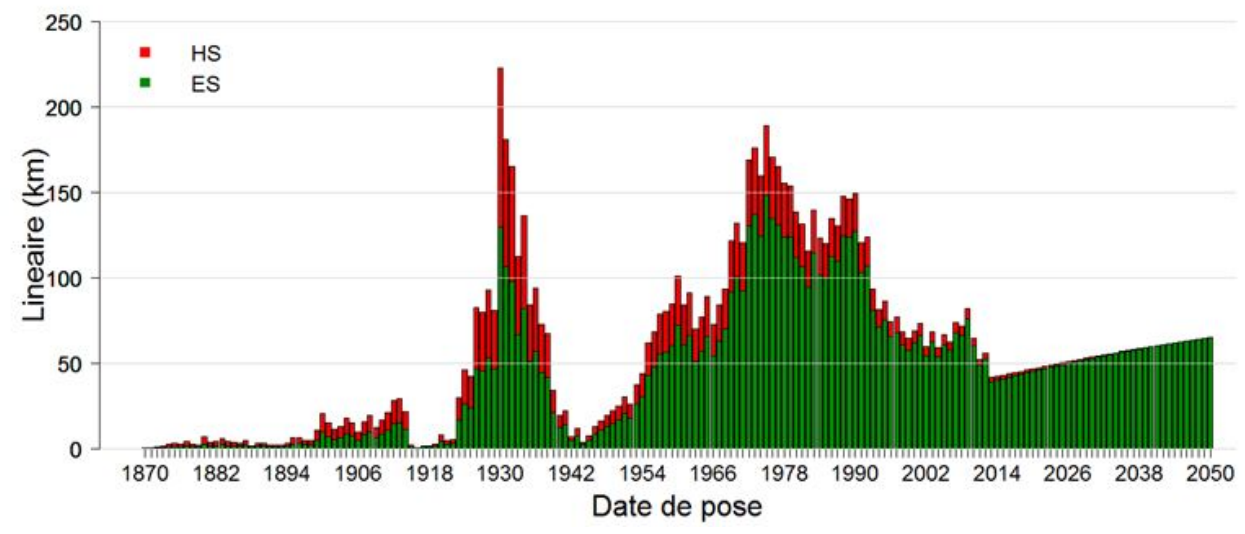

Figure 3. Distribution par date de pose $\left(y_{0}\right)$ du linéaire en service (ES) en 2050 et mis hors service (HS) entre 2012 et 2050 au SEDIF.

La figure 4 présente l'indicateur IP1 et IP2 sur le territoire d'eauservice Lausanne avec le scénario statu quo stratifié par matériau.
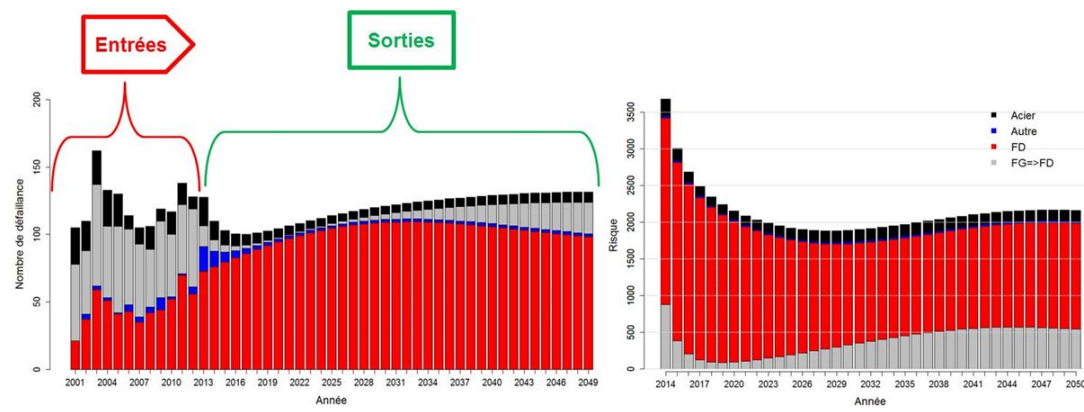

Figure 4. Nombre de défaillances passées et prévues (IIP) (à gauche) et valeur de l'indicateur de risque de dommage liée à une coupure d'eau induite par des défaillances (I2P) (à droite) pour le réseau d'eauservice Lausanne stratifié par matériau.

Afin de valider l'ordre de grandeur de ces onze indicateurs, les valeurs prospectives de ces indicateurs sont systématiquement comparées aux valeurs passées de ces même indicateurs.

\section{Conclusions et perspectives}

Après un important travail de consolidation des données et d'analyse des pratiques, la mise en œuvre du modèle long terme OPTIMEAU, reposant sur l'exploitation de courbes de survie rétrospectives, a permis d'obtenir de premiers résultats prometteurs. Le modèle OPTIMEAU est original : - l'utilisation des courbes de survie passées propres à chacun des services d'eau permet d'introduire dans le modèle des pratiques réalistes et non arbitraires ; - la construction innovante de l'indicateur prévisionnel du nombre de défaillances futures repose sur la modélisation des défaillances observées et intègre les effets du renouvellement à venir. Le modèle OPTIMEAU permet de simuler les effet d'une stratégie sur l'évolution future de onze indicateurs (de moyens, de résultats et de performance), permettant aux gestionnaires de juger si cette stratégie est cohérente avec leurs objectifs. Par exemple des indicateurs de coûts du renouvellement prévus, de risque etc. sont calculés. De plus l'ensemble des tronçons peuvent être stratifiés selon des variables pertinentes regroupées en classes comme le type de matériau, le diamètre ou la localisation; ce qui permet d'établir des courbes de survie par strate et d'obtenir des indicateurs (linéaire, coûts, etc.) plus précis. Cette démarche est très reproductible et est applicable sans problème sur des réseaux de 
différentes tailles. Elle a été appliquée avec succès sur le réseau d'eau de la ville de Lausanne en Suisse (900km) ou sur celui du SEDIF 9 fois plus étendu.

L'enjeu majeur de notre travail qui consiste, sur la base de données réelles, à établir par la modélisation un lien entre modèles de court terme et modèles de long terme est ainsi satisfait. Les améliorations en cours visent à s'écarter de la stratégie statu quo en recherchant des stratégies optimales. Ce travail devra se faire en deux étapes. Dans un premier temps, à partir d'une analyse fine des processus opérationnels de décision « court terme ", il sera étudié comment les diverses causes de mise hors service des tronçons contribuent à la construction des courbes de survie observées. Dans un second temps, il sera possible de reconstruire de nouvelles courbes de survie opérationnelles prospectives, qui intégreront de manière explicite les éléments essentiels qui président au processus de décision (par exemple le pourcentage des travaux pilotés par le vieillissement $v s$ le pourcentage piloté par le renouvellement de la voirie). On pourra ainsi estimer comment des stratégies différentes (par exemple profiter plus largement des opportunités de travaux) modifient les performances de long terme.

\section{Remerciements}

L'auteure remercie l'IRSTEA et l'Université de Bordeaux pour avoir porté le projet. Merci également à eauservice Lausanne, au Grand Lyon, au SEDIF, au fonds de recherche pour l'eau de la Société Suisse de l'Industrie du Gaz et des Eaux et aux agences de l'eau (Rhône Méditerranée, Seine Normandie) pour l'aide technique et financière qu'ils apportent dans la thèse.

\section{Bibliographie}

[AWW 10] AWWA, Buried no longer : confronting america's water infrastructure challenge, rapport, American Water Works Association (AWWA), 2010.

[CAD 02] CADOR J., Le renouvellement du patrimoine en canalisations d'eau potable en France, rapport, Univ. Caen, 2002.

[CHE 07] Chevassus Aulouis B., L'analyse des risques - L'expert, le décideur et le citoyen., QUAE, Versailles, 2007.

[DEQ 14] Dequesne J., Brejoux E., Liccardi A., Claverolas-Renard A., Observatoire des services publics d'eau et d'assainissement. Panorama des services et de leur performance en 2010, rapport, ONEMA, 2014.

[ENG 05] Engelhardt M. O., Skipworth P. J., «WiLCO, State of the art decision support», Proceedings of the 8th International Conference on Computing and Control for the water Industry, Exeter, United Kingdom, p. 27-32, 2005.

[FuC 08] Fuchs-Hanusch D., Gangl G., Kornberger B., Kölbl J., Hofrichter J., Kainz H., « PiReM, Pipe rehabilitation management developing a decision support system for rehabilitation planning of water mains », Water Practice and Technology, vol. 3(1), $\mathrm{n}^{\circ}$ 1, page 9, 2008.

[HER 02] HERZ R., «Developing rehab strategies for drinking water networks, Dresden University of Technology, Germany », Proceedings of the 9 DBMC International Conference on Durability of Building Materials and Components, page 11, 2002.

[JAR 01] JARRETT R., Hussain O., VeEvers A., VAN DER Touw J., «A review of asset management models available for water pipeline networks », Proceedings of International Conference of Maintenance Societies ICOMS, Melbourne, 2001.

[KAP 58] KAPLAN E. L., MEIER P., « Non parametric estimation from incomplete observations. », Journal of the American Statistical Association, vol. 53, $\mathrm{n}^{\circ}$ 282, p. 457-481, 1958.

[KRO 13] KROPP I., Kanew software, version 4, manual., 3S Consult GmbH, 2013.

[LAR 13] LARGE A., Optimisation du renouvellement des canalisations, Rapport de première année de thèse : revue bibliographique et cadre méthodologique, rapport, IRSTEA et Université de Bordeaux, 2013.

[LEG 13] LE GAT Y., «Extending the Yule Process to model recurrent pipe failures in water supply networks », Urban Water Journal, vol. published on line, page 15, 2013.

[MAR 10] MARKOV A. A., «Investigation of the general case of trials associated into a chain (In Russian). », Actes de la Société physico-mathématique, Université de St Pétersbourg, 8e série., vol. 25, page 33, 1910.

[MAR 09] Marlow D., Urquharta A., Davis P., Trans D., Beale D., Burn S., Remaining Asset Life : A State of the Art Review, IWA publishing, 2009.

[NAL 13] NALDEO, Patrimoine expert, Outil de simulation du vieillissement du patrimoine canalisations AEP, Manuel de l'utilisateur, Version 3.4, Naldeo ingénierie et conseil, 2013.

[OIE 12] OIEAU, E\&Y, Etude de la récupération des coûts des services liés à l'utilisation de l'eau pour les bassins hydrographiques français en application de la directive cadre sur l'eau, rapport, OIEau et Ernst \& Young, 2012.

[SAE 03] Saegrov S., König A., Mattick A., Milina J., Röstum J., Selseth I., « Methods for estimating water network rehabilitation needs. », Water Supply, vol. 3, p. 63-69, 2003.

[WEI 51] WeIBUlL W., «A statistical distribution function of wide applicability », Journal of Applied Mechanics ASME, vol. 18, p. 293-297, 1951. 